\title{
Anaesthetic management of siblings having xeroderma pigmentosum: a rare presentation
}

\begin{abstract}
Xeroderma Pigmentosum (XP) is a rare autosomal recessive disease which causes skin pigmentation with precancerous lesions and neurological abnormalities. These manifestations are due to a cellular hypersensitivity to ultraviolet (UV) radiation leading to a defect in repair of DNA by the process of nucleotide excision repair (NER) mechanism. Here, we are reporting a child with XP scheduled for excision of basal cell carcinoma over the central area of scalp under general anaesthesia.
\end{abstract}

Keywords: xeroderma pigmentosum, mac coy laryngoscope, dexmedetomidine, papulonodular, ulcerative, tracheal intubation
Volume 4 Issue 2 - 2016

\author{
Alok Kumar Bharti, Neeraj Kumar, Arun \\ Kumar AM, Bikram Kumar Gupta, Saurabh \\ suman \\ Department of neuroanaesthesiology and critical care All India
} Institute of Medical Sciences, India

\author{
Correspondence: Neeraj Kumar, Department of \\ Neuroanaesthesiology and critical care, All India Institute of \\ Medical Sciences, New Delhi, India, Tel 8505864856, \\ Email neeraj.jInmc@gmail.com
}

Received: October 24, 2015 | Published: January 18, 2016

\section{Introduction}

A xeroderma pigmentosum is a rare hereditary autosomal recessive disorder and presence of basal cell carcinoma in children is rare. ${ }^{1}$ It is mainly characterized by hypersensitivity to sunlight, ocular involvement, and progressive neurological complications. ${ }^{2}$ These manifestations are due to defect in repair of DNA by the process of nucleotide excision repair (NER) mechanism caused by ultraviolet radiation. ${ }^{2}$ As a xeroderma pigmentosum induced changes are mainly dermatological, it leads to several challenges in anaesthetic management. Here, we are reporting a child with a diagnosed case of Xeroderma pigmentosum associated with basal cell carcinoma situated over the central part of the scalp posted for a wide excision and a superficial skin grafting under general anaesthesia.

\section{Case report}

A 8 years old male, weighing $17 \mathrm{~kg}$ body weight with ASA grade I presented with a diagnosed case of Xeroderma pigmentosum associated with basal cell carcinoma situated over the central part of the scalp posted for a wide excision and a superficial skin grafting under general anaesthesia. He was having xeroderma pigmentosum since 5 years back; he presented with recurrent swelling at multiple sites mainly involving both upper and lower eye lids, tip of the nose and extended over the forehead (Figure 1). From the age of 2 years dermatological manifestation in the form of papulonodular ulcerative indurated lesion with pearly transparent rim lying over the central part of the scalp were present which gradually increasing in severity and size and measuring $8 \mathrm{~cm} \times 5.0 \mathrm{~cm} 2$ (Figure 2). A multiple actinic keratosis poikiloderma was present in areas which are especially exposed to ultraviolet radiation. On exposure to sunlight child mainly complained of watering from both eyes with photophobia and occasionally associated with recurrent allergic conjunctivitis. General examinations were unremarkable. Another interesting fact in our report is that his elder sibling a ten years old child also suffered from the similar disease presented with recurrent dermatological lesion over the scalp which later on diagnosed as basal cell carcinoma (Figure 3). Her elder sibling has a past history of multiple surgeries for basal cell carcinoma of nose, forehead and lateral canthus of eye and he already received two cycles of chemotherapy regimen including inj. 5 FU $500 \mathrm{mg}$ and inj. cisplatin $30 \mathrm{mg}$. The elder sibling was under regular follow up for his chemotherapy regimen. The preanaesthetic evaluation of the child was done in an environment with low light. On proper airway assessment: Upper lip bite test were done for temporomandibular mobility assessment it was grade II (Lower incisor can partially hide the mucosa of upper lip) and Inter incisor distance was more than $1.5 \mathrm{~cm}$. Thyromental distance $>6 \mathrm{~cm}$ and mallampatti class III were present. No restricted neck movements were present. On physical examination following specific findings were present like bilateral corneal clouding, narrow opening of both external nares and a large tongue. A multiple irregular nodular actinic keratosis poikiloderma present throughout the body, especially in areas exposed to ultraviolet radiation. Systemic examinations were normal. All routine investigation was unremarkable. So based on above findings a difficult airway (including both mask ventilation and tracheal intubation) were anticipated in view of numerous skin nodules over face and large tongue. A written and informed consent were taken from his mother for emergency Tracheostomy in view of anticipated difficult airway apart from difficult airway cart were kept ready. For anxiolysis tab alprazolam $0.25 \mathrm{mg}$ and as antacid prophylaxis tab ranitidine $150 \mathrm{mg}$ were advised a night before surgery. NPO orders were issued. As premedication, thirty minutes prior to procedure oral midazolam suspension $(10 \mathrm{mg})$ were given to keep the child quiet and cooperative. On arrival to the operation theatre all standard monitoring were attached in the form of ECG, NIBP, SPO2, ETCO2 and axillary skin temperature probe. On preoxygenation a proper child face mask fitting were not possible because of multiple skin nodules over the face and smaller external nare openings. So paediatric face masks were used over the child mouth for securing proper seal and adequate mask ventilation. Then the child was induced with sevoflurane on increasing concentration till child maintain spontaneous respiration, meanwhile venous access was established in right lower limb on multiple attempts as it was difficult to obtain venous access in both upper extremities due to skin changes. On obtaining intravenous access injection fentanyl $2 \mu \mathrm{g} / \mathrm{kg}$ body weight were given. Then anaesthesia was maintained with dexmedetomidine $(30 \mu \mathrm{g})$ infused over $10 \mathrm{~min}$, and the child were under continuous monitoring as there was an increased risk for excessive sedation leading to airway compromise. After placing the child in a sniffing position, first we performed conventional laryngoscopy with Macintosh blade 2 and it was difficult to visualize 
the epiglottis. So Mac coy laryngoscope were used as an alternative for performing laryngoscopy on second attempt and posterior one third of vocal cord were visualised with difficulty but on applying BURP manueveur endotracheal intubation were performed using 6 $\mathrm{mm}$ ID cuffed endotracheal tube over a gum elastic bougie. Tracheal tube placement was confirmed by capnography and bilateral air entry on auscultation. As non depolarizing muscle relaxant atracurium was used as a dose of $0.5 \mathrm{mg} / \mathrm{kg}$. Intraoperative period of surgery and anaesthesia were uneventful. On 5 th postoperative day the child were discharged with further follow up for chemotherapy regimen.

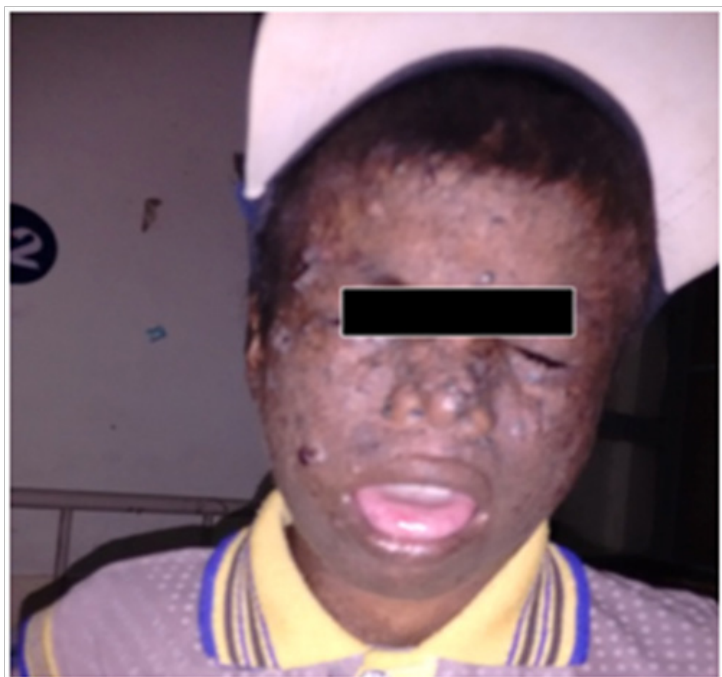

Figure I Younger child having Xeroderma Pigmentosum.

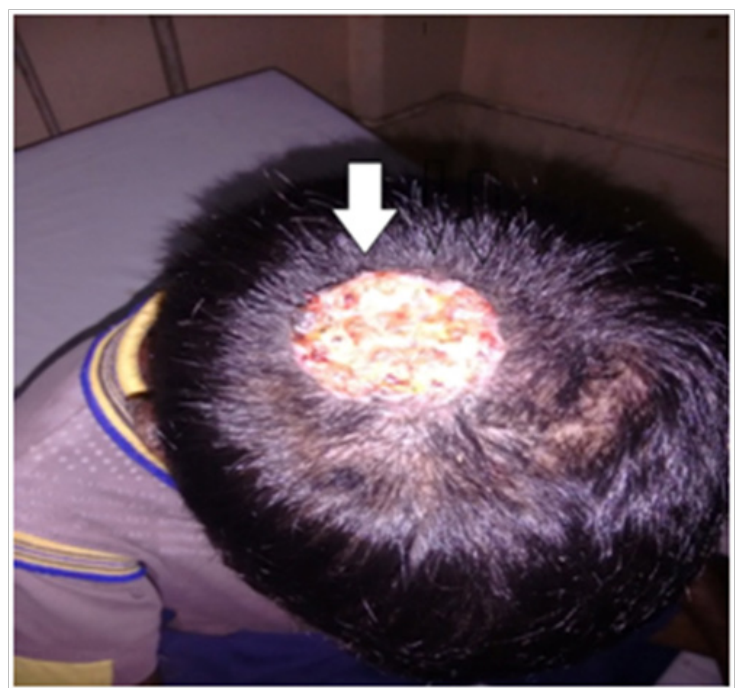

Figure 2 Showing Basal cell carcinoma lesion over central part of scalp.

\section{Discussion}

Several dermatological manifestations in the form of keratosis, pigmented skin, severe sunburn, marked freckle ,persistent erythema, keratosis, telangiectasis, and neoplasm were present in the xeroderma pigmentosum patients imposed several challenges to the anaesthesia. Xeroderma pigmentosum patient will develop skin cancer by 8 years of age if associated with multiple primary lesions. ${ }^{2}$ The multiple facial skin nodules leading to improper fitting of the face mask in our case resulting in difficulty during mask ventilation. Another challenging situation was difficulty in tracheal intubation due to presence of large tongue and limited mouth opening. We were aware of the potential airway difficulty, so supraglottic airway devices and cricothyroid and retrograde intubation were set up apart from emergency tracheostomy, but as mask ventilation was not possible using standard face mask, a paediatric face mask were used for mouth ventilation. The endotracheal intubation were performed in the second attempt with the help of gum elastic bougie and a McCoy laryngoscope using BURP (Backward, upward, right ward and posterior) manueveur as conventional laryngoscopy was not successful using Macintosh laryngoscope. The McCoy levering laryngoscope has been well recognized as a useful tool for certain cases of difficult intubation. ${ }^{3,4}$ Using an inhalation induction along with dexmedetomidine as infusion for sedation, we were able to maintain spontaneous breathing in our patient, enabling us to safely secure the airway without compromise. A nasotracheal intubation was avoided in our case because of limited external nare openings. As far as our knowledge is concern this is the first case report suggesting successful management of xeroderma pigmentosum using a $\alpha 2$ agonist dexmedetomidine in a child. The nitrous oxide was avoided as he was on 5-Flurouracil. However, according to report by Miyazaki et al..$^{5}$ a TIVA technique, using propofol and fentanyl was described in xeroderma pigmentosum patients.A study by Masuda et al. ${ }^{6}$ avoided nitrous oxide because 5-Fluorouracil can cause myelosuppression and avoided inhalational agents such as halogenated anaesthetics cause genotoxic effects and deranged NER in cells. A study by Oliveira et al. Showed that minimum usage of muscle relaxants under the monitoring of neuromuscular block is done in patients of XP due to neuronal dysfunction, skin atrophy, and joint contracture. ${ }^{7}$ As inhalational agent sevoflurane mainly used for its favourable properties suitable for paediatric anaesthesia and for short procedures. ${ }^{8}$ An ultraviolet light found in sunlight damages genetic material (DNA) in skin cells. Normally, the body repairs this damage but in persons with $\mathrm{XP}$, the body does not fix the damage due to molecular defects in genes involved in NER. ${ }^{2}$ The most common causes of death in XP subjects are due to skin cancer, neurological degeneration, or internal cancer. ${ }^{8}$ As a DNA injury is cumulative and irreparable so a definitive treatment of XP is still not well established. Prophylaxis is based primarily on the patient's protection from sunlight, through the use of sun screens, sunglasses and clothes that prevent the penetration of ultraviolet rays. ${ }^{9}$ Surgery and electro coagulation, as well as the use of 5- fluorouracil, retinoids and immunotherapy are few treatment options are available, but none is completely effective. ${ }^{10-14}$

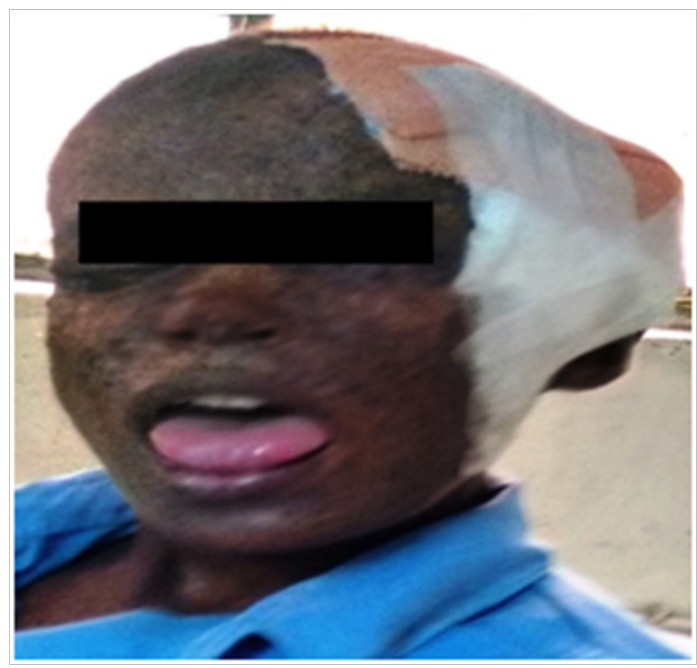

Figure 3 Elder sibling having Xeroderma pigmentosum. 
Most important anaesthetic concerns mainly related to dark pigmented lesions in the form of multiple actinic keratosis which poses a great challenges during both mask ventilation and endotracheal intubation and difficulty in obtaining intravenous cannulation and fixation, even a repeated exposure to surgery and anaesthesia put a huge sociological and psychological impact especially in a child and their relatives. Proper eye care, padding of the pressure point and hypothermia are also a important anaesthetic concerns especially if xeroderma pigmentosum is present in child. We successfully managed the anticipated difficult mask ventilation and intubation using paediatric face mask for mouth ventilation and Mac Coy laryngoscopy with BURP manueveur for performing anticipated difficult intubation along with dexmedetomidine for maintenance infusion and as non depolarizing agent atracurium were used in a minimal dose.

\section{Conclusion}

Patients suffering from xeroderma pigmentosum need proper anaesthetic management with no harmful drugs and protective covering to skin with meticulous care. Dermatological manifestation especially around face and oropharynx caused by this pathology poses great challenges for facial mask adaptation and tracheal intubation A proper psychological and social support to the patient and their families along with constant education should be the sole and primary objective in the management of Xeroderma pigmentosum.

\section{Acknowledgments}

Published with the written consent of patient parent.

\section{Authorship contribution}

i. Alok Kumar Bharti: Concept, Analysis of concept, final approval.

ii. Neeraj Kumar Dube: Drafting the article, writing the paper, final approval.

iii. Atchaya Arun Kumar: Analysis of concept.

iv. Bikram kumar Gupta: Analysis of concept, final approval.

v. Saurabh suman: Final approval.

\section{Conclusion}

Emergency airway management at any site is lifesaving for the moribund patients in clinical practice. Although the consequences are not so optimistic with pre-existing irreversible massive organ damage instead of following all prescribed protocols meticulously. But, the rescue measures unfortunately may become sometimes life- threatening due to improper assessment, actions and futile over-try in difficult scenarios. Moreover, off-theatre circumstances are often revealed quite odd and unfamiliar to the caregivers. Therefore, proactive preparedness, early detection and referral, correct decisions, purposeful actions and trained assistance are solicited for better outcomes.

\section{References}

1. LeSueur BW, Silvis NG, Hansen RC. Basal cell carcinoma in children: Report of 3 cases. Arch Dermatol. 2000;136(3):370-372.

2. Feller L, Wood NH, Motswaledi MH, et al. Xeroderma pigmentosum: A case report and review of the literature. J Prev Med Hyg. 2010;51(2):87-91.

3. Tuckey JP, Cook TM, Render CA. Forum. An evaluation of the levering laryngoscope. Anaesthesia. 1996;51(1):71-73.

4. Aoyama K, Nagaoka E, Takenaka I, et al. The McCoy laryngoscope expands The laryngeal aperture in patients with difficult intubation. Anesthesiology. 2000;92(6):1855-1856.

5. Miyazaki R, Nagata T, Kai T, et al. Anesthesia for a patient with xeroderma pigmentosum. Masui. 2007;56(4):439-441.

6. Masuda Y, Imaizumi H, Okanuma M, et al. Anesthesia for a patient with xeroderma pigmentosum. Masui. 2002;51(2):169-171.

7. Oliveira CR, Elias L, Barros AC, et al. Anesthesia in patient with Xeroderma Pigmentosum: Case report. Rev Bras Anestesiol. 2003;53(1):46-51

8. Lehmann AR, McGibbon D, Stefanini M. Xeroderma pigmentosum. Orphanet J Rare Dis. 2007;6:70

9. Nalgirkar AR, Borkar SS, Nalgikar AS. Xeroderma pigmentosum with multiple malignances. Indian Pediatrics. 2000;37:1377-1379.

10. Candiani OJ, Siwady GS, Gutierrez LF, et al. Dermabrasion in xeroderma pigmentosum. Dermatol Surg. 1996;22(6):575-577.

11. Craven NM, Griffiths CF. Retinoids in the management of nonmelanoma skin cancer and melanoma. Cancer Surv. 1996;26:267-288.

12. Saade M, Debahy NE. Xeroderma pigmentosum Clinical remission of squamous cell carcinoma-associated with isotretinoin and chemotherapy: case report. J Chemother. 1977;11:313-317.

13. Wee SY, Ahn DS. Facial resurfacing Xeroderma pigmentosum in with chemical peeling. Plast Reconstr Surg. 1999;103(5):1464-1467.

14. Shah PJ, Dubey KP, Yadav JP. Predictive value of upper lip bite test and ratio of height to Thyromental distance compared to other multivariate airway assessment test for difficult laryngoscopy in apparently normal patient. J Anaesthesio Clin Pharmacol. 2013;29(2):191-195. 